УДК 616.8:159.942.32:616-091

Для цитирования: Скутин А.В., Ефименко Т.С., Шайхетдинов Р.Г., Громов В.А. Проводящие пути и нейроанатомические корреляты симптоматического (патологического), нормального (эмоционального) и произвольно вызванного смеха (обзор зарубежной литературы). Сибирский вестник психиатрии и наркологии. $2018 ; 2$ (99): $111-119$. https://doi.org/10.26617/1810-3111-2018-2(99)-111-119

\title{
Проводящие пути и нейроанатомические корреляты симптоматического (патологического), нормального (эмоционального) и произвольно вызванного смеха (обзор зарубежной литературы)
}

\author{
Скутин А.В. ${ }^{1}$, Ефименко Т.С. ${ }^{2}$, Шайхетдинов Р.Г. ${ }^{3}$, Громов В.А. ${ }^{3}$ \\ ${ }^{1}$ Уральский государственный университет физической культуры \\ Россия, 454091, Челябинск, ул. Орджоникидзе, 1 \\ ${ }^{2}$ Южно-Уральский государственный медичинский университет \\ Россия, 454076, Челябинск, ул. Воровского, 64 \\ ${ }^{3}$ Южно-Уральский государственный университет (Национальный исследовательский университет) \\ Россия, 454080, Челябинск, пр. Ленина, 76
}

\section{PEЗЮME}

Используя классические методы неврологии, были определены и каталогизированы области мозга, связанные с симптоматическим (патологическим) смехом, по другим диагностическим признакам и симптомам таких состояний, как эпилепсия, инсульты и поражения головного мозга, выявленные в результате осмотра. Эти наблюдения были дополнены новыми исследованиями, использующими современные неинвазивные методы. В обзоре также обсуждаются недавние исследования, посвященные вопросам, связанным с «нормальным» и произвольно вызванным смехом, а также формулируется структура нейроанатомических коррелятов смеха на основе исследований лабораторных животных, неврологических пациентов и нормальных испытуемых, которые рассматриваются в настоящем труде.

Ключевые слова: симптоматический смех, спонтанный смех, произвольно вызванный смех, проводящие пути, нейроанатомические корреляты.

\section{ВВЕДЕНИЕ}

То, что смех и юмор являются неотъемлемыми компонентами человечества, вряд ли нужно документировать; их анализировали и обсуждали более двух тысячелетий, традиционно в контексте философии, антропологии, психологии и филологии [36, 58]. С XIX века патологические варианты смеха заинтересовали неврологов [15, 69]. Однако прошло более 20 лет с того, как был опубликован последний крупный обзор в этой области [75]. Недавно «нормальный» смех также попал в сферу исследования неврологии [39, 45, 70]. Разумеется, нормальный смех может быть вызван другими элементами, кроме юмора, такими как щекотка, индуцированный смех и веселящий газ $[62,77,78]$. Однако в настоящее время юмор является единственным элементом, используемым для того, чтобы вызвать нормальный смех в неврологических исследованиях. Чарльз Дарвин в книге «Выражение эмоций в человеке и животных» (1872) предположил, что эволюционная основа смеха была его функцией в социальном выражении счастья, и это обеспечило совместное преимущество выживания для социума [25]. Улыбка и смех не уникальны для людей. Среди молодых особей шимпанзе отмечено, что «игровое лицо» с соответствующей вокализацией сопровождает игру, щекотание или игровой укус [76, 98].

\section{ОБСУЖДЕНИЕ ПРОБЛЕМЫ}

У людей ответная улыбка развивается в течение первых 5 недель внеутробной жизни [52]. Смех появляется позже, около четвертого месяца [81]. Хотя на морфологическом уровне выделено более 16 типов улыбок [28], интересно, что разные типы смеха (в юмористических ситуациях, но также презрительные, насмешливые, социальные, поддельные и т.д.) остаются относительно необозначенными [81]. Улыбка в ответ на юмор - это конфигурация лица [29], обозначающая «улыбку Дюшена», которая относится к одновременному сокращению большой скуловой мышцы и орбикулярной мышцы (которые вытягивают углы губ назад и вверх и сужают глаза, вызывая морщины). Во время смеха активируются дополнительные лицевые, дыхательные и гортанные мышцы [6, 81]. Улыбка и смех могут возникать спонтанно (в ответ на юмор, эмоциональные или социологические стимулы), а также могут быть вызваны по команде (произвольная или «фальшивая» улыбка/смех).

Патологический смех. Изучение симптоматического смеха десятилетиями предшествовало изучению нормального смеха. Существуют разные определения патологического смеха $[24,85]$, но наиболее часто упоминаемое определение относится к определению Пёка [74]. По его критериям, патологический смех - это смех, который возникает: (1) в ответ на неспецифические стимулы; (2) при отсутствии соответствующего изменения эмоциональной реакции; (3) при отсутствии добровольного контроля над масштабом или продолжительностью эпизода; (4) при отсутствии соответствующего изменения настроения, продолжающегося за пределами уже произошедшего смеха. 
На протяжении многих лет другие условия для состояний, в которых происходит патологический смех, включают «непроизвольный смех», «аффективную лабильность» [63], «фиктивное веселье» [59], «неуместный», «неуправляемый» и «неэпилептический» смех, «эмоциональное недержание» [50]. Признанной схемой классификации симптоматического смеха является схема Пёка $[74,75]$. Что касается невропатологии, он дифференцировал симптоматический смех исходя из: (1) бокового амиотрофического склероза, сосудистого псевдобульбарного паралича и экстрапирамидных двигательных расстройств; (2) fou rire prodromique (продрома сумасшедшего смеха); (3) эпилептических припадков.

Эпилепсия смеха. Смех может встречаться в рамках эпилептического припадка. Термин «эпилепсия смеха» (от греч. gelotos, смех) относится к относительно редким судорогам, в которых смех является основным симптомом. Эти приступы могут состоять исключительно из смеха, но часто возникают в связи с вегетативным возбуждением и непроизвольными движениями и/или нарушенными состояниями сознания $[10,19,21,88,97,103]$. Сообщалось о симптоме, сопровождающем иктальный смех, таком как мочеиспускание, однако он встречается реже [90]. Несмотря на стереотипный характер, смех пациентов во время припадков эпилептического смеха может казаться нормальным и даже быть заразным; один из таких пациентов даже выиграл конкурс «счастливый ребенок» [10]. Смех при эпилепсии является механическим и неестественным $[11,90]$. Иктальная улыбка (без смеха) наблюдалась у пациентов с эпилептическими очагами в затылочно-теменной, гиппокампиальной и височной областях [64]. Сообщалось об эпилептическом смехе при генерализованном туберозном склерозе [88].

Гипоталамические гамартомы. Исследование с помощью однофотонной эмиссионной компьютерной томографии продемонстрировало состояние гиперперфузии в этих опухолях во время припадков эпилептического смеха [5]. Во время припадков выделяются гормоны гипоталамуса и гипофиза [5, 21, 93]. Предположение, что гипоталамус ответствен за возникновение этих приступов подтверждено тремя наблюдениями: 1) электрическая стимуляция гамартом вызывает типичные приступы [54]; 2) в отношении биохимии гипоталамических гамартом магнитно-резонансная спектроскопия показала снижение отношения $\mathrm{N}$-ацетил-аспарагиновой кислоты и креатина в области опухоли, но не в прилегающих областях мозга [59, 90]. Снижение пика $\mathrm{N}$-ацетиласпарагиновой кислоты считается признаком дегенерации нейронов, но не обязательно указывает на патологические изменения, а может отражать изменчивость спектральной картины между анатомическими формациями из-за неоднородности их гистоморфологии; 3) хирургическое удаление опухоли может снизить частоту приступов [54, 68, 72, 96, 97]. Опухоли обладают возбуждающими эффектами, причём аномальная электрическая активность рас- пространяется рострально и дорзально в соседней лимбической системе и каудально к мозговому стволу, что способствует психофизиологическим проявлениям «смеха» [54].

Fou rire prodromique (продрома сумасшедшего смеха) (Фере, 1903) - редкое состояние, когда немотивированный, неуместный смех возникает как первый симптом церебральной ишемии [31]. Этот смех, кажущийся неконтролируемым, может сопровождаться хихиканьем [100] или плачем [7], а затем заменяется симптомами инсульта: гемипарез или афазия [74]. Смех fou rire prodromique описывается как «громкий и сердечный» [7] и как имеющий «хихикающий» характер [74] и может продолжаться до 30 минут [100]. Повреждения найдены: (1) у основания моста [100], (2) в левой извилине гиппокампа, левой заднелатеральной части таламуса и прилегающих частях внутренней части капсулы [20], (3) в левых чечевицеобразном и хвостатом ядрах с участием передней островковой доли большого мозга [18], (4) в области, снабжаемой правой средней мозговой артерией [55]. Смех в этих случаях вызван поражениями двигательных нейронов. Однако короткое возбуждающее действие ишемии не может быть исключено [66]. Большинство сообщаемых феноменов fou rire prodromique свидетельствуют о сосудистых кровоизлияниях.

Патологический смех из-за других неврологических расстройств. Большинство публикаций о смехе при неврологических расстройствах с синдромами неуместной и неконтролируемой улыбки или смеха, которые происходят хронически. Уилсон (1924) описал случаи патологического смеха, например, у больного, который после двух инсультов «каким бы ни был эмоциональный стимул, и каким бы лёгким он ни был ... начинал смеяться ... громко [103]. Прочитав военные новости, он начинал улыбаться, и чем серьёзнее была новость, тем больше он смеялся». У пациента с «рассеянным склерозом» Уилсон сообщил, что «взрывы длинного, неуправляемого, но почти бесшумного смеха происходили из-за ... мелочей». Патологический смех обычно не соответствует ситуации, в которой возникает [24]. Пациент может осознавать эту несостоятельность, но бессилен контролировать смех $[87,89]$. Неуместный смех часто вызван тривиальными стимулами [85]. Смех может резко измениться до плача [74]. Однако патологический смех понимается как расстройство двигательных сопутствующих состояний, аффективных выражений, включающих респираторные, вазомоторные, секреторные и вокальные компоненты. Нейрофизиологическое исследование патологического и нормального смеха у 6 пациентов, проведенное Танака и Сумицудзи [89], обнаружило, что при патологическом смехе наблюдались сокращения лобных и бровных мышц; то есть пациенты хмурились и в то же время смеялись, тем самым придавая своим лицам «напряженное», а не «расслабленное» выражение. Неясно, были ли сокращения лба попытками контролировать движе- 
ние лица добровольно или они были связаны с переходом от улыбки к смеху, который у обычных наблюдаемых также часто включает сокращения лба [81].

Патологический смех включает в себя ритмические клонические движения диафрагмы, развивающиеся не по нарастанию, как нормальный смех, а внезапно. В связи со сложностью данной проблематики представляют научный интерес шкалы интенсивности эмоциональных выражений лица, разработанные Слоэном и его коллегами, а также Робинсоном и его коллегами [79, 87]. Имеются данные, что патологический смех зависит от серотонинергической и дофаминергической передачи, поскольку сообщалось о положительных результатах лечения у пациентов, получавших селективные блокаторы обратного захвата серотонина $[61,73,83,87$, 95]. Патологический смех связан с поражениями головного мозга, обнаруженными в областях от лобной коры и пирамидального тракта до дорсального отдела мезенцефалона и моста. Нейрофизиологическое действие большинства этих поражений, по-видимому, связано с хроническим растормаживанием вышеозначенного пути.

В одном из первых исследований патологического смеха (и плача) Уилсон (1924) описал пациентов с опухолями в покрышке и верхней части моста [103]. В последнее время опубликованы сообщения о пациентах с вентрально локализованными поражениями головного мозга, производящими безрадостный смех. Бхатхивале и коллеги сообщили о 4 пациентах с компрессией моста (из-за тройничных невром) [12], а Мутон и коллеги и Тей и Сакамото о 2 пациентах с сосудистыми инсультами, у одного в правой ножке мозга, мосте и каудальном отделе мезенцефалона, у другого - в вентромедиальном отделе моста [65, 91]. Подобные симптомы проявлялись у пациента с понтомедуллярной глиомой [56], пациента с хордомой ската (оказывающей давление на понтомезенцефалические структуры в вентральном направлении) [60] и пациента с каменистоскатовой менингиомой [85]. В исследовании 49 пациентов с парамедиальными инфарктами моста головного мозга на основании МРТ и магнитнорезонансной ангиографии Катаока и коллеги дифференцировали пациентов с инфарктами в (1) парамедиальной базальной, (2) парамедиальной тегментальной областях [49]. Лишь 3 пациента в первой группе проявляли патологический смех, все они страдали дизартрией, 2 из них - лицевым парезом.

Стриатокапсулярные области. Несмотря на высокую частоту стриатокапсулярных инфарктов, сообщения о патологическом смехе у этих пациентов относительно редки. 3 пациента с такими инфарктами (2 больших по площади и 1 маленький) демонстрировали спонтанные безрадостные атаки смеха во время речи, стресса или фрустрации, которые начинались и заканчивались внезапно [20]. Другие сообщения включают исследования Ким и Чой-Квон (2000) [50], Пёка (1969) [74] и Арлазарова и др.
(1998) [3].

Поражения лобной доли. Мендез и коллеги описали пациента, страдающего постоянным патологическим смехом, по-видимому, из-за бифронтальной медиальной энцефаломаляции (после разрыва аневризмы) с бифронтальным гипометаболизмом в ПЭТ-исследовании [63]. Патологический смех являлся симптомом у $10 \%$ пациентов с рассеянным склерозом, причем рост частоты происходит параллельно с прогрессированием заболевания [30]. Патологический смех наблюдался при боковом амиотрофическом склерозе [37, 61], хронической прогрессирующей спинобульбарной спастичности [67] и прогрессирующей супрануклеарной дегенерации двигательной системы [101]. Сакеим и коллеги в обзоре 119 опубликованных случаев обнаружили преобладание правосторонних поражений, связанных с патологическим смехом [82]. Другие исследования не подтвердили это латеральное доминирование [50].

Таким образом, мезенцефалические или мостовые поражения, связанные с патологическим смехом, были в вентральных областях вышеперечисленных структур. Патологический смех в результате поражений лобной доли или поражений в стриатокапсулярной области регистрировался редко.

Диссочиаџия произвольного смеха и ульюки. Парезы произвольных выражений лица могут возникать, когда выражение лица, обусловленное эмоциями, безмятежно. Это состояние названо синдромом Фуа-Шавани-Мари, «передним оперкулярным синдромом» или «произвольным парезом лицевых мышц» [43]. Возможна обратная ситуация: парез эмоционально стимулированных лицевых мышц может возникать в то время, как произвольно контролируемые выражения лица неизменны, как при парезе эмоционально стимулированных лицевых мышц [43] и амимии [48]. Типичные поражения, приводящие к произвольному парезу лицевых мышц, располагаются билатерально в покрышке и могут возникать врождённо либо у взрослых в результате сосудистых инсультов, опухолей [14]. В синдроме Фуа-Шавани-Мари присутствуют тяжёлая дизартрия и парезы дистальных черепных нервов [101]. Произвольные парезы лицевых нервов наблюдались у пациентов с инфарктами левой средней мозговой артерии (с сохранением таламостриальных ветвей) [41] или двигательной коры и от частичных инфарктов лучистого венца [43]. Это состояние связано с поражениями внутренней капсулы [44], парамедиальной области моста без участия тегментума [94] и большими поражениями в лобнотеменной части белого вещества и сообщалось у пациента с рассеянным склерозом и крупными вентроцентральными поражениями белого вещества [43]. Подводя итог, необходимо отметить, что все эти поражения были расположены в премоторных областях (передней части покрышки) или вдоль кортикобульбарных двигательных путей. Из-за этих повреждений могут возникать произвольные парезы 
лицевого нерва и патологический смех. Когда патологический смех сопровождается произвольным парезом лицевого нерва, обусловленные повреждения обычно были множественными, подкорковыми и располагались в вентральной части мезенцефалона либо в мосте. Очевидно, что не все пациенты с повреждениями кортикобульбарных двигательных путей страдают от патологического смеха.

Смех, веселье и стимуляиия мозга. За последние 20 лет в зарубежной хирургии стало обычной практикой стимулировать открытую поверхность мозга электрически, пытаясь найти эпилептогенные очаги. Первый такой случай сообщили Фиш и коллеги [32]. Улыбка была вызвана у двух из 75 пациентов, мозг которых стимулировался. Во время этих эпизодов улыбки мозг стимулировался в миндалевидной (у одного пациента) либо в лобной коре (у другого). К сожалению, помимо мест стимулирования областей отсутствуют сведения о характере вызванных улыбок. Подобные случаи вызванной улыбки сообщались Гордоном и коллегами [40], два из 106 пациентов проявляли смех или улыбались, когда их мозг стимулировался. Этих же пациентов подробно описывали Арройо и коллеги, сообщавшие о проявлении смеха, связанного с весельем, когда стимулировалась веретеновидная извилина или извилина гиппокампа [4]. Эти пациенты сообщали, что во время стимуляции «значимость вещей менялась» и что все было «смешно», а также о чувствах счастья и головокружения. В двух письмах к научным журналам $[9,53]$ и в одной статье [51] описаны анекдотические сообщения о «чувстве благополучия» (связанном со смехом и весельем) во время стимуляции субталамического ядра у 6 пациентов с болезнью Паркинсона. У 1 пациента с гамартомой стимуляция гипоталамуса вызывала смех [54]. По более старым источникам, индукция смеха сообщается в связи с электрической стимуляцией в передней коре головного мозга [84] и бледном шаре [42]. Фрид и коллеги описали поведение и субъективные чувства молодой пациентки, которая начала смеяться после того, как её мозг стимулировали в левой верхней лобной извилине [35]. Стимуляция других областей мозга в непосредственной близости вызвала заторможенность речи и движения рук.

Изучение нечеловеческих смехоподобных вокализаций. Хотя смех, связанный с юмором, кажется феноменом, уникальным для людей, существуют поведенческие модели эмоционально вызванных вокализаций у других приматов и даже у крыс, которые имеют сходство с социальным смехом. Степень, в которой недавно сообщённое «пищание» крыс (Панксепп, 2000), вызванное щекоткой, поведенчески гомологично смеху (возможность, которую предлагает автор), требует дальнейшей этиологической оценки [71].

Нейроанатомия смеха. Сообщения показывают, что в области мезенцефалона и моста существует функциональное разделение между структурами, необходимыми для формирования эмоционально обусловленных выражений, с одной стороны, и произвольных, эмоционально нейтральных выражений - с другой. Вентральные поражения в этих областях приводят к парезам произвольно созданных мимических выражений без повреждений либо с преувеличенным выражением эмоций. Поражения в дорзальных, тегментальных структурах приводят к подавлению эмоционального выражения. Для анатомических областей, ростральных к мезенцефалону, это разделение не так ясно: поражения базальных ядер или внутренней капсулы могут быть связаны с патологическим смехом, парезом произвольных мышц или эмоциональных парезов. Что касается повреждений в таламусе, сообщалось об эмоциональных состояниях, но нет сообщений о парезах произвольных мышц и патологическом смехе или плаче. Патологический смех, с другой стороны (но не эмоциональные парезы), был связан с обширными лобными поражениями головного мозга, поражениями в мозжечке и дегенеративными заболеваниями трактов, протекающих от двигательной и премоторной коры головного мозга.

Уилсон (1924) оказал влияние на развитие этой области за последние десятилетия [103]. Он отметил, что в смехе (и плаче) участвуют мимические мышцы и мышцы, участвующие в дыхательном и голосовом контроле. Он подчеркнул, что таламус не обязательно включается в контроль над смехом, в отличие от общего консенсуса, существовавшего со времени работы Нотнагеля (1889) [69]. Дэвисон и Кельман (1939) предположили, что гипоталамус и другие диэнцефальные ядра, таламические центры, стриатум и бледный шар участвуют в производстве аффективных реакций [26]. Мартин (1950) первым подчеркнул значение гипоталамуса в этих процессах и предположил, что центр смеха находится в гипоталамусе или рядом с ним (на основании исследования 4 пациентов, среди которых один был исследован при аутопсии) [57]. Он придумал фразу «фиктивное веселье» (по аналогии с фиктивной яростью) для эмоциональных выражений, проявляющихся во время эпилепсии смеха.

Пёк (1969) предположил (как и Уилсон) «центр координации» смеха [74]. По Пёку, патологический смех происходит не в результате повреждений пирамидального тракта, а только тогда, когда такие поражения сосуществуют с подкорковыми нарушениями в кортикоретикулярных трактах. Пёк опроверг модель Уилсона с её произвольной и непроизвольной иннервацией. Тогда патологический смех мог возникнуть из любого из четырёх уровней: (1) поражения внутренней части капсулы с участием базальных ядер; (2) поражения чёрной субстанции в связи с другими экстрапирамидными поражениями; (3) поражения каудального гипоталамуса; (4) двустороннего повреждения пирамидного тракта.

Браун (1967) полагал, что всё связано со стволом мозга [16] и предложил механизм синхронизации в ростральном отделе среднего мозга, ответственном за координацию смеха, плача и ярости. Он 
предположил, исходя из данных пациентов и экспериментов на животных, что: (1) центральную роль играет ретрансляция связи между нисходящим лимбико-диэнцефалическим трактом и бульбарными эффекторными органами, интегрируя приток из разных областей (гиппокамп, дорсомедиальная часть таламуса, латеральные части гипоталамуса посредством медиального переднего пучка), а также восходящие ретикулярные и спиноталамические связи в вентральную и парамедиальную области головного мозга; (2) ретикулярная формация, активируясь, вызывает смех (или плач, связанный с дыханием, мимикой и вокализацией); (3) мезэнцефалическое центральное серое вещество через аннулооливарный тракт к мозжечку может оказывать модулирующее воздействие, изменяя тональность смеха. В свете анатомических связей от серого вещества и ретикулярной формации к церебральным областям, как показано в экспериментах на животных, кажется более вероятным, что приток из разрознённых областей мозга (гипоталамус, лобная кора, базальная область височной коры, базальные ядра, таламус) может быть достаточным, чтобы вызвать реакцию, составляющую смех $[8,92,99]$. Особая роль гипоталамуса также вероятна, учитывая данные пациентов с эпилепсией смеха из-за гипоталамических гамартром и из экспериментов на животных $[1,99]$. Возможность того, что мозжечок играет определённую роль в модуляции и координации этих процессов, должна оставаться под вопросом. Однако представляется возможным, что на основе синаптических связей у нормальных людей мозжечок может быть вовлечен в эмоциональное выражение.

Юмор и мозг. Отношения между субъективными чувствами эмоций (возбуждением) и его двигательными выражениями (улыбкой и смехом) обсуждались более века [46] и остаются предметом оживленного дискурса [23]. Поскольку описанные ниже эксперименты основаны на одной из этих теорий, теории несоответствий Канта [47] - теория превосходства Платона и Аристотеля [2] и психоаналитическая теория Фрейда [34] в данном обзоре не обсуждались. По теории несоответствий, юмор включает восприятие несоответствия или парадокса в игривом контексте [33]. Для чего-то смешного в обработке юмористического материала выделено два этапа. На первом этапе «...воспринимающий не находит подтверждения своего ожидания в отношении текста в окончании шутки... получатель сталкивается с несоответствием - кульминацией». На втором этапе воспринимающий участвует в форме решения проблем, чтобы найти когнитивное правило, согласно которому кульминация вытекает из основной части шутки и примиряет несоответствующие части. Другие исследователи назвали эти стадии «неожиданностью» и «связностью» [17]. Для некоторых психологов эти два этапа недостаточны для объяснения различий между восприятием юмора и аналогичной ситуацией, восприятием того, что проблема решена. Высказано предположение, что двухэтапную модель следует расширить, включив третий этап [80]: выявление того, что на самом деле имеет смысл (учитывая способность воспринимать юмор), является приятной глупостью. Дагге и Хартье (1985) изучали пациентов с повреждением правого полушария [22]. Нарушения в понимании мультфильмов в большей степени связаны с дефицитом в их зрительно-перцептивной и когнитивной способности, чем с их неспособностью идентифицировать аффективные компоненты мультфильмов. Бирле и коллеги сообщили о подобных результатах при использовании мультфильмов и вербальных шуток, тем самым добавив поддержку гипотезы о том, что правое полушарие играет особую роль в процессах, необходимых для понимания юмора, независимо от способа восприятия, при котором представлен юмористический материал [13]. Шамми и Штусс исследовали 21 пациента с однократным очаговым поражением правого полушария головного мозга, ограниченным лобной (правой, левой или бифронтально) или нефронтальной (правой или левой) областями [86]. Пациенты с лобными поражениями правого полушария показали наибольшие нарушения в способности отличать юмористические от неюмористических мультфильмов и реагировали с меньшей физической или эмоциональной реактивностью (смех, улыбка).

Изучение юмора у нормальных субъектов. В исследовании электрической активности коры, связанной с обработкой информации юмора, Деркс и коллеги сообщили о пике активности в событийнообусловленных потенциалах (ERP) 300 мс после прослушивания кульминации шутки [27]. За этим позже последовала общая деполяризация 100 мс. Предполагается, что две волны параллельны двухступенчатой когнитивной модели обработки юмора. [33]. Показано, что на обработку юмора может влиять настроение: положительное настроение по сравнению с негативным сопровождалось большими различиями в ERP между шутками, вызывающими смех, и теми, которые его не вызывали. В двух последних исследованиях функциональная МРТ использовалась для демонстрации областей кислородно-зависимой церебральной активности у нормальных субъектов, когда они слушали шутки. В первом из них (Озава и др., 2000) у 10 прослушивавших запись трёх жанров текстов в рамках использования функционального аппарата МРТ: шутки, газетную статью и философский текст [70]. Позже субъекты оценивали отдельные тексты в отношении того, насколько смешным был текст и насколько трудно было понять его. В соответствии с лингвистическим характером задач область Вернике и поперечные височные извилины (билатерально) активировались у всех субъектов во всех условиях. Предложения, которые испытуемые оценили как забавные, индуцировали активацию в области Брока и средних лобных извилинах (соответствующих синтаксической обработке и слуховой памяти); те, которые считались трудными для понимания, связаны с ак- 
тивностью в левой нижней париетальной доле (соответствующей семантической обработке) и задней части левой верхней височной извилины.

Во втором исследовании (Гоэль и Долан, 2001) 14 субъектам были представлены фонологические шутки (каламбуры) и семантические шутки (основанные на юморе, отличном от простой языковой игры) [39]. Пока они находились в функциональном сканере МРТ, субъекты делали суждения (записанные с помощью нажатия клавиши) относительно того, были ли им интересны те или иные истории; после сканирования субъекты рассматривали шутки и оценивали их по шкале забавности. Кортикальная активация, связанная с прослушиванием каламбуров, обнаружена в левой задней средней височной и левой нижней лобной извилинах, тогда как активность, связанная с прослушиванием семантических шуток, обнаружена в левой задней средней височной, левой задней нижней височной и правой задней средней височной извилинах и мозжечке. Церебральная активность в медиальной вентральной префронтальной коре параллельно изменялась с оценками субъектами забавности шутки и может быть связана с аффективной оценкой юмора.

В обоих исследованиях восприятие шуточно обусловленного юмора связано с кислороднозависимой активностью крови в височных областях и левых лобных областях, но области в двух исследованиях не совпадали в точности, а области деятельности описаны во втором исследовании и не представлены в первом. Ни одно исследование не включало контроль над такими искажающими факторами, как внимание или эмоциональные реакции на лице. Предположение о том, что юмор является причиной наблюдаемых активаций, может быть преждевременным. Резюмируя результаты по юмору и мозгу, необходимо отметить, что есть убедительные доказательства из исследований пациентов с поражением головного мозга, что для восприятия юмора необходимо недоминирующее полушарие и что его лобные области особенно важны $[38,86]$. Когда юмор воспринимался обычными, нормальными испытуемыми, эти области активно не проявлялись $[39,45,70]$. Когда смех или веселье индуцировались электрофизиологически у пациентов с эпилепсией (которые противопоставлялись нормальным), были вовлечены префронтальная кора [35] и базальные части височных долей [4]. Что касается последних исследований в области изображения у нормальных субъектов, то в областях мозга, связанных с обработкой юмора, были включены средняя лобная извилина и область Брока [70], медиальная передняя префронтальная кора, левая нижняя лобная, левая задняя средняя и нижняя височная извилины и мозжечок [39].

\section{ЗАКЛЮЧЕНИЕ}

Выражение смеха, по-видимому, зависит от двух частично независимых проводящих нейронных путей. Первый - «непроизвольный»/спонтанный, или «эмоционально управляемый». Данная система включает области миндалевидного тела, таламические/гипо- и субталамические области и дорсальный отдел ствола мозга. Вторая - «произвольная» (искусственно вызванный смех) система - возникает в премоторных/фронтальных оперкулярных областях головного мозга и ведёт через моторную область коры и пирамидальный тракт к вентральному отделу ствола мозга. Эти системы и реакция смеха, по-видимому, согласовываются координационным центром смеха в дорзальной части моста.

«Аномальные реакции смеха» (патологический смех) могут инициироваться поражениями на трёх уровнях: (1) фасциально-дыхательных бульбарных ядер и надсегментных двигательных путей; (2) диэнцефальный и лимбический уровень; (3) гипоталамический, лобный и височный уровень, где, теоретически, возникают психические расстройства, в том числе настроения и когнитивных функций.

Во время здоровых эмоциональных реакций (смех, плач, нахмуривание) серое вещество головного мозга и верхняя часть ретикулярной формации получают возбуждающий приток из префронтальной или базальной височной коры и базальных ядер и гипоталамуса. Есть предположение, что эти реакции подвергаются произвольному влиянию (вероятно, тормозящих) трактов, идущих от премоторной и двигательной коры, через церебральные ножки, к вентральной стороне ствола мозга. Пока неясно, как на этом уровне мозга нейронные действия изменяются, когда они связаны с эмоциями (веселье, горе, удивление). Естественно, многие выражения лица могут быть сформированы произвольно; однако большинство людей не могут убедительно подражать подлинным выражениям лица испытываемых эмоций. Это особенно сложно в связи со смехом. Таким образом, существует предположение, что подлинный, эмоционально производный смех не начинается в двигательной коре, а скорее всего, что во время такого смеха прекращается торможение кортикального лобного отдела. Также рассматривается появление патологического смеха как результат повреждения тормозящей системы. Представляется возможным, что у пациентов с вентрально располагающимися опухолями ствола головного мозга вызванное давлением разрушение тормозящих путей приводит к принудительной мимике [102].

\section{КОНФЛИКТ ИНТЕРЕСОВ}

Авторы заявляют об отсутствии конфликта интересов в связи с публикацией данной статьи.

\section{ИСТОЧНИК ФИНАНСИРОВАНИЯ}

Авторы заявляют об отсутствии финансирования при проведении исследования.

ЛИТЕРАТУРА/REFERENCES

1. Abrahamson E.E., Moore R.Y. The posterior hypothalamic area: chemoarchitecture and afferent connections. Brain Res. 2001; 889: 1-22.

2. Aristotle A. Nicomachean ethics. In: McKeon R., editor. The basic works of Aristotle. New York: Random House; 1941.

3. Arlazaroff A., Mester R., Spivak B., Klein C., Toren P. Pathological laughter: common vs. unusual aetiology and presentation. Isr J Psychiatry Relat Sci. 1998; 35: 184-189.

4. Arroyo S., Lesser R.P., Gordon B. et al. Mirth, laughter and 
gelastic seizures. Brain, 1993; 116: 757-780.

5. Arroyo S., Santamaria J., Sanmarti F. et al. Ictal laughter associated with paroxysmal hypothalamopituitary dysfunction. Epilepsia. 1997; 38: 114-117.

6. Bachorowski J.A., Smoski M.J., Owen M.J. The acoustic features of human laughter. J Acoust Soc Am. 2001; 110: 15811597.

7. Badt B. Lachen als erstes Symptom eines apoplektischen Insultes. Z ges Neurol Psychiat. 1927; 110: 297-300

8. Bandler R., Keay K.A. Columnar organization in the midbrain periaqueductal gray and the integration of emotional expression. Prog Brain Res. 1996; 107: 285-300.

9. Beijjani B.P., Damier P., Agid Y. Transient acute depression induced by high-frequency deep-brain stimulation. New Engl J Med. 1999; 341: 1004.

10. Berkovic S.F., Andermann F, Melanson D. et al. Hypothalamic hamartomas and ictal laughter: evolution of a characteristic epileptic syndrome and diagnostic value of magnetic resonance imaging. Ann Neurol. 1988; 23: 429-439.

11. Berkovic S.F., Kuzniecky R.I., Andermann F. Human epileptogenesis and hypothalamic hamartomas: new lessons from an experiment of nature. Epilepsia. 1997; 38: 1-3.

12. Bhatjiwale M.G., Goel A., Desai K. Pathological laughter as a presenting symptom of trigeminal neurinoma-case report. $\mathrm{Neu}$ rol Med Chir Tokyo. 1996; 36: 644-646.

13. Bihrle A.M., Brownell H.H., Powelson J.A., Gardner H. Comprehension of humorous and nonhumorous materials by left and right brain damaged patients. Brain Cogn. 1986; 5: 399-411.

14. Bingham P.M., Parrish B., Chen S.C. et al. Spectrum of disorders associated with enlarged sylvian fissures in infancy. $\mathrm{Neu}$ rolog. 1998; 51: 1732-1735.

15. Brissaud E. Lezons sur les maladies nerveuseus. Paris: Masson; $1895 ; 446-468$

16. Brown J.W. Physiology and phylogenesis of emotional expression. Brain Res. 1967; 5: 1-14.

17. Brownell H.H., Michel D., Powelson J., Gardner H. Surprise but not coherence: sensitivity to verbal humor in right hemisphere patients. Brain Lang. 1983; 18: 20-27.

18. Carrell A.T. Humor communities. Humor. 1997; 10: 11-24.

19. Cascino G..D., Andermann F., Berkovic S.F. et al. Gelastic seizures and hypothalamic hamartomas: evaluation of patients undergoing chronic intracranial EEG monitoring and outcome of surgical treatment. Neurology. 1993; 43: 747-750.

20. Ceccaldi M., Milandre L. A transient fit of laughter as the inaugural symptom of capsular-thalamic infarction. Neurology. 1994; 44: 1762

21. Cerullo A., Tinuper P., Provini F. et al. Autonomic and hormonal ictal changes in gelastic seizures from hypothalamic hamartomas. Electroencephalogr Clin Neurophysiol. 1998; 07: 317-322.

22. Dagge M., Hartje W. Influence of contextual complexity on the processing of cartoons by patients with unilateral lesions. Cortex. 1985; 21: 607-616.

23. Damasio A. Looking for Spinoza: joy, sorrow, and the human brain. New York: Harcourt; 2003. 27-80.

24. Dark F.L., McGrath J.J., Ron M.A. Pathological laughing and crying. Australian and New Zealand Journal of Psychiatry. 1996; 30: 472-479.

25. Darwin C. The expression of the emotions in man and animals. London: John Murray; 1872.

26. Davison C., Kelman H. Pathological laughter and crying. Arch Neurol Psychiat. 1939; 42: 595-643.

27. Derks P., Gillkin L.S., Bartolome-Rull D.S., Bogart E.H. Laughter and electroencephalographic activity. Humour. 1997. 10: 285-300.

28. Ekman P. What we have learned by measuring facial behavior Ekman P., Rosenberg E.L., editors. What the face reveals. New York: Oxford University Press; 1997: 469-485.

29. Ekman P., Davidson R.J., Friesen W.V. The Duchenne smile: emotional expression and brain physiology: II. J Pers Soc Psychol. 1990; 58: 342-353.

30. Feinstein A., Feinstein K., Gray T., O'Connor P. Prevalence and neurobehavioral correlates of pathological laughing and crying in multiple sclerosis. Arch Neurol. 1997; 54: 1116-1121.

31. Fere M.C. Le fou rire prodromique. Rev Neurol (Paris). 1903; 11: 353-358.
32. Fish D.R., Gloor P., Quesney F.L., Olivier A. Clinical responses to electrical brain stimulation of the temporal and frontal lobes in patients with epilepsy. Pathophysiological implications. Brain. 1993; 116: 397-414.

33. Forabosco G. Cognitive aspects of the humor process: the concept of incongruity. Humor. 1992; 5: 45-68.

34. Freud S. Jokes and their relation to the unconscious. Translated by J. Strachey. Harmondsworth: UK: Penguin; 1976.

35. Fried I., Wilson C.L., MacDonald K.A., Behnke E.J. Electric current stimulates laughter. Nature. 1998; 391: 650.

36. Fry W. Humor and the brain: a selective review. Humor. 2002; 15: $305-333$.

37. Gallagher J.P. Pathologic laughter and crying in ALS: a search for their origin. Acta Neurol Scand. 1989; 80: 114-117.

38. Gardner H., Ling P.K., Flamm L., Silverman J. Comprehension and appreciation of humorous material following brain damage. Brain. 1975; 98: 399-412.

39. Goel V., Dolan R.J. The functional anatomy of humor: segregating cognitive and affective components. Nat Neurosci. 2001; 4: 237-238.

40. Gordon B., Hart J. Jr., Lesser R.P., Arroyo S. Mapping cerebral sites for emotion and emotional expression with direct cortical electrical stimulation and seizure discharges. Prog Brain Res. 1996; 107: 617-622

41. Gschwend J. The dissociated laughing and weeping in apoplectic insult. Fortschr Neurol Psychiatr Grenzgeb. 1978; 46: 629-632.

42. Hassler R., Riechert T. Wirkungen der Reizungen und Koagulationen in den Stammganglien bei stereotaktischen Hirnoperationen. Nervenarzt. 1961; 32: 97-109.

43. Hopf H.C., Muller-Forell W., Hopf N.J. Localization of emotional and volitional facial paresis. Neurology. 1992; 42: 19181923.

44. Husain A.M. Neurological picture. Mimetic smile. J Neurol Neurosurg Psychiatry. 1997; 63: 144.

45. Iwase M., Ouchi Y., Okada H. et al. Neural substrates of human facial expression of pleasant emotion induced by comic films: a PET study. Neuroimage. 2002; 17: 758-768.

46. James W. The principles of psychology. 3rd ed. New York: Dover; 1950.

47. Kant I. Kant's Critique of judgement. Translated by J.H. Bernard. New York: Hafner, 1972.

48. Karnosh L.J. Amimia or emotional paralysis of the face. Dis Nerv Syst, 1945; 6: 106-108

49. Kataoka S., Hori A., Shirakawa T., Hirose G. Paramedian pontine infarction. Neurological/topographical correlation. Stroke. 1997; 28: 809-815.

50. Kim J.S., Choi-Kwon KS. Poststroke depression and emotional incontinence: correlation with lesion location. Neurology. 2000; 54: 1805-1810.

51. Krack P., Kumar R., Ardouin C. et al. Mirthful laughter induced by subthalamic nucleus stimulation. Mov Disord. 2001; 16: 867-875.

52. Kraemer M., Abrahamsson M., Sjostrom A. The neonatal development of the light flash visual evoked potential. Doc Ophthalmol. 1999; 99: 21-39.

53. Kumar R., Krack P., Pollak P. Transient acute depression induced by high-frequency deep-brain stimulation. New Engl J Med. 1999; 341: 1003-1004.

54. Kuzniecky R., Guthrie B., Mountz J. et al. Intrinsic epileptogenesis of hypothalamic hamartomas in gelastic epilepsy. Ann Neurol. 1997; 42: 60-67.

55. Lago A. Fou rire prodromique and ischemic stroke. Stroke. 1998; 29: 1067-1068.

56. Lal A.P., Chandy M.J. Pathological laughter and brain stem glioma. J Neurol Neurosurg Psychiatry. 1992; 55: 628-629.

57. Martin J.P. Fits of laughter (sham mirth) in organic cerebral disease. Brain. 1950; 70: 453-464.

58. Martin R. Approaches to the sense of humor: a historical review. In: Ruch W., editor. The sense of humor. Berlin: Mouton de Gruyter, 1998: 16-60.

59. Martin D.D., Seeger U., Ranke M.B., Grodd W. MR imaging and spectroscopy of a tuber cinereum hamartoma: a patient with growth hormone deficiency and hypogonadotropic hypogonadism. AJNR Am J Neuroradiol. 2003; 24: 1177-1180.

60. Matsuoka S., Yokota A., Yasukouchi H. et al. Clival chordoma associated with pathological laughter. Case report. J Neurosurg. 
1993; 79: 428-433.

61. McCullagh S., Feinstein A. Treatment of pathological affect: variability of response for laughter and crying. J Neuropsychiatry Clin. Neurosci. 2000; 12: 100-102.

62. McGhee P.E. Humor: its origin and development. San Francisco: W.H. Freeman; 1979.

63. Mendez M.F., Nakawatase T.V., Brown C.V. Involuntary laughter and inappropriate hilarity. $J$ Neuropsychiatry Clin. Neurosci. 1999; 11: 253-258.

64. Molinuevo J.L., Arroyo S. Ictal smile. Epilepsia. 1998; 39: 1357-1360

65. Mouton P., Remy A., Cambion H. Spasmodic laughter caused by unilateral involvement of the brain stem. Rev Neurol (Paris). 1994; 150: 302-303.

66. Nardone R., Tezzon F. Inhibitory and excitatory circuits of cerebral cortex after ischaemic stroke: prognostic value of the transcranial magnetic stimulation. Electromyogr Clin Neurophysiol. 2002; 42: 131-136.

67. Nishimura M., Tojima M., Suga M. et al. Chronic progressive spinobulbar spasticity with disturbance of voluntary eyelid closure. Report of a case with special reference to MRI and electrophysiological findings. J Neurol Sci. 1990; 96: 183-190.

68. Nishio S., Morioka T., Fukui M., Goto Y. Surgical treatment of intractable seizures due to hypothalamic hamartoma. Epilepsia. 1994; 35: 514-519.

69. Nothnagel H. Zur Diagnose der Sehhgelerkrankungen. Z Klin Med. 1889; 16: 424-430.

70. Ozawa F., Matsuo K., Kato C. et al. The effects of listening comprehension of various genres of literature on response in the linguistic area: an fMRI study. Neuroreport. 2000; 11: 11411143.

71. Panksepp J., Burgdorf J. 50-kHz chirping (laughter) in response to conditioned and unconditioned tickle-induced reward in rats: effects of social housing and genetic variables. Behav Brain Res. 2000; 115: 25-38.

72. Parrent A.G. Stereotactic radiofrequency ablation for the treatment of gelastic seizures associated with hypothalamic hamartoma. Case report. J Neurosurg. 1999; 91: 881-884.

73. Parvizi J., Anderson S.W., Martin C.O. et al. Pathological laughter and crying. A link to the cerebellum. Brain. 2001; 124: 1708-1719.

74. Poeck K. Pathophysiology of emotional disorders associated with brain damage. In: Vinken P.J., Bruyn G.W., editors. Handbook of clinical neurology. V. 3. Amsterdam: Elsevier: 1969. 343-367.

75. Poeck K. Pathological laughter and crying. In: Frederik S., editor. Handbook of clinical neurology. V. 1. Amsterdam: Elsevier, 1985: 219-225.

76. Preuschoft S. 'Laughter' and 'smiling' in macaques - an evolutionary perspective. Utrecht: University of Utrecht Press, 1995.

77. Provine R.R. Laughter: a scientific investigation. New York: Viking, 2000: 23-53, 158-162.

78. Ramachandran V.S., Blakeslee S. Phantoms in the brain. New York: William Morrow, 1998: 208.

79. Robinson R.G., Parikh R.M., Lipsey J.R., Starkstein S.E., Price T.R. Pathological laughing and crying following stroke: validation of a measurement scale and a double-blind treatment study. Am J Psychiatry. 1993; 150: 286-293.

80. Ruch W., Hehl F.-J. A two-mode model of humor appreciation: its relation to aesthetic appreciation and simplicity-omplexity of personality. In: Ruch W. (ed.) The sense of humor: explorations of a personality characteristic. Berlin: Mouton de Gryter, 1998: 109-142.

81. Ruch W., Ekman P. The expressive pattern of laughter. In: Kaszniak A., editor. Emotion, qualia and consciousness. Tokyo: World Scientific, 2001: 426-443.

82. Sackeim H.A., Greenberg M.S., Weiman A.L. et al. Hemispheric asymmetry in the expression of positive and negative emotions. Neurologic evidence. Arch Neurol. 1982; 39: 210-218.

83. Seliger G.M., Hornstein A., Flax J. et al. Fluoxetine improves emotional incontinence. Brain Inj. 1992; 6: 267-270.

84. Sem-Jacobsen C.W. Depth-electrographic stimulation of the human brain and behavior: from fourteen years of studies and treatment of Parkinson's disease and mental disorders with implanted electrodes. American lecture series, publication No. 710. Springfield (IL): Charles C. Thomas, 1968.

85. Shafqat S., Elkind M.S., Chiocca E.A. et al. Petroclival meningioma presenting with pathological laughter. Neurology. 1998; 50: 1918-1919.

86. Shammi P., Stuss D.T. Humour appreciation: a role of the right frontal lobe. Brain. 1999; 122: 657-666.

87. Sloan R.L., Brown K.W., Pentland B. Fluoxetine as a treatment for emotional lability after brain injury. Brain Inj. 1992; 6: 315319.

88. Striano S., Meo R., Bilo L. et al. Gelastic epilepsy: symptomatic and cryptogenic cases. Epilepsia. 1999; 40: 294-302.

89. Tanaka M., Sumitsuji N. Electromyographic study of facial expressions during pathological laughing and crying. Electromyogr Clin Neurophysiol. 1991; 31: 399-406.

90. Tasch E., Cendes F., Li L.M. et al. Hypothalamic hamartomas and gelastic epilepsy: a spectroscopic study. Neurology. 1998; 51: 1046-1050.

91. Tei H., Sakamoto Y. Pontine infarction due to basilar artery stenosis presenting as pathological laughter. Neuroradiology. 1997; 39: 190-191.

92. Ter Horst G.J., Copray J.C., Liem R.S., Van Willigen J.D. Projections from the rostral parvocellular reticular formation to pontine and medullary nuclei in the rat: involvement in autonomic regulation and orofacial motor control. Neuroscience. 1991; 40: 735-758.

93. Tinuper P., Cerullo A., Rosati A., Cortelli P. Case of a child with gelastic seizures and hypothalamic hamartoma. Epilepsia. 1997; 38: 1363-1364.

94. Trepel M., Weller M., Dichgans J., Petersen D. Voluntary facial palsy with a pontine lesion. J. Neurol. Neurosurg. Psychiatry. 1996; 61: 531-533

95. Udaka F., Yamao S., Nagata H. et al. Pathologic laughing and crying treated with levodopa. Arch Neurol. 1984; 41: 10951096.

96. Unger F., Schrottner O., Haselsberger K. et al. Gamma knife radiosurgery for hypothalamic hamartomas in patients with medically intractable epilepsy and precocious puberty. Report of two cases. J Neurosurg. 2000; 92: 726-731.

97. Valdueza J.M., Cristante L., Dammann O. et al. Hypothalamic hamartomas: with special reference to gelastic epilepsy and surgery. Neurosurgery. 1994; 34: 949-958.

98. Van Hoof J.A. A comparative approach to the phylogeny of laughter and smiling. In: Hinde R.A. editor. Non-verbal communication. London: Cambridge University Press; 1972: 209-241.

99. Veazey R.B., Amaral D.G., Cowan W.M. The morphology and connections of the posterior hypothalamus in the cynomolgus monkey (Macaca fascicularis). II. Efferent connections. J Comp Neurol. 1982; 207: 135-156.

100. Wali G..M. 'Fou rire prodromique' heralding a brainstem stroke. J Neurol Neurosurg Psychiatry. 1993; 56: 209-210.

101. Weller M. Anterior opercular cortex lesions cause dissociated lower cranial nerve palsies and anarthria but no aphasia: FoixChavany-Marie syndrome and 'automatic voluntary dissociation' revisited. J Neurol. 1993; 240: 199-208.

102. Wild B., Rodden F., Grodd W., Ruch W. Neural correlates of laughter and humor. Brain Article history Views. 2003; 126 (10): 2121-2138.

103. Wilson S.A.K. Some problems in neurology, No. II. Pathological laughing and crying. J Neurol. Psychopath. 1924; 4: 299333.

Поступила в редакцию 23.01.2018

Утверждена к печати 2.04.2018

Скутин Андрей Викторович, к.м.н., доцент кафедры спортивной медицины и физической реабилитации. Ефименко Татьяна Сергеевна, к.м.н., доцент кафедры клинической психологии и социальной работы. Шайхетдинов Рашит Гильметтинович, ст. преподаватель кафедры физического воспитания.

Громов Виктор Александрович, к.п.н., доцент кафедры физического воспитания. 
Скутин Андрей Викторович, andrey.sckutin@yandex.ru УДК 616.8:159.942.32:616-091

For citation: Skutin A.V., Efimenko T.S., Shaikhetdinov R.G., Gromov V.A. Pathways and neuroanatomical correlates of symptomatic (pathological), normal (emotional) and voluntarily caused laughter (review of foreign literature). Siberian Herald of Psychiatry and Addiction Psychiatry. 2018; 2 (99): 111-119. https://doi.org/10.26617/1810-3111-2018-2(99)-111-119

\section{Pathways and neuroanatomical correlates of symptomatic (pathological), normal (emotional) and voluntarily caused laughter (review of foreign literature)}

\section{Skutin A.V. ${ }^{1}$, Efimenko T.S. ${ }^{2}$, Shaikhetdinov R.G. ${ }^{3}$, Gromov V.A. ${ }^{3}$}

${ }^{1}$ Ural State University of Physical Culture

Ordzhonikidze Street 1, 454091, Chelyabinsk, Russian Federation

${ }^{2}$ South Ural State Medical University

Vorovsky Street 64, 454076, Chelyabinsk, Russian Federation

${ }^{3}$ South Ural State University (National Research University)

Lenin Avenue 76, 454080, Chelyabinsk, Russian Federation

\section{ABSTRACT}

Using classical methods of neurology, areas of the brain associated with symptomatic (pathological) laughter were identified and cataloged according to other diagnostic signs and symptoms of such conditions as epilepsy, strokes and brain lesions detected as a result of the examination. These observations were supplemented by new studies using modern non-invasive methods. The review also discussed recent studies on issues related to "normal laughter" and also formulated the structure of neural correlates of laughter and humor based on studies of laboratory animals, neurological patients and normal subjects, which were discussed in this work.

Keywords: symptomatic laughter, spontaneous laughter, randomly generated laughter, pathways, neuroanatomical correlates.

Skutin Andrey V., PhD, Associate Professor of Sports Medicine and Physical Rehabilitation Department, Ural State University of Physical Culture, Chelyabinsk, Russian Federation.

Efimenko Tatiana S., PhD, Associate Professor of Clinical Psychology and Social Work Department, South Ural State Medical University, Chelyabinsk, Russian Federation.

Shaikhetdinov Rashit G., senior lecturer of the Department of Physical Education, South Ural State University (National Research University), Chelyabinsk, Russian Federation.

Gromov Viktor A., PhD, Associate Professor of the Department of Physical Education, South Ural State University (National Research University), Chelyabinsk, Russian Federation.

Skutin Andrey V., andrey.sckutin@yandex.ru 